\title{
Les surfaces cuticulaires des gnathopodes de deux Crustacés Amphipodes: Niphargus (Gammaridé hypogé) et Gammarus (Gammaridé épigé)
}

\author{
par
}

\author{
Evelyne SELLEM*
}

Un traitement au nitrate d'argent vital appliqué à certains Amphipodes Talitridés (Orchestia) et Gammaridés (Niphargus) permet de mettre en évidence des aires cuticulaires particulières sur leurs gnathopodes 1 (Gn1) et 2 (Gn2) (Magniez, 1966). Complété par l'action de leuco-dérivés et par la réaction de von Kossa au nitrate d'argent, ces régions squelettiques s'avèrent être perméables non seulement aux ions argent mais également à l'oxygène et ne sont pas calcifiées. Elles se comportent vis à vis des différents réactifs utilisés de la même façon que les branchies (Graf et Magniez, 1969). Ces surfaces présentent en outre une très fine ornementation, ornementation remarquée dans la région sterno-distale du propodite du deuxième gnathopode des individus indifférenciés et des $\uparrow$ d'Orchestia gammarella (Pallas) par Charniaux-Cotton en 1957 et décrite comme étant constituée d'une multitude "de petites pointes". Nous avons été conduit à préciser la localisation et la configuration de ces plages ornementées chez les ở et les $q$ de cinq espèces d'Orchestia (Graf et Sellem, 1970; Sellem, 1971). Elles sont toutes très sensibles au nitrate d'argent vital et situées sur la face sternale du propodite, du carpopodite et du méropodite des Gn1 et Gn2, mais les gnathopodes présentent, chez Orchestia, un fort dimorphisme sexuel ce qui entraîne des modifications dans la répartition des plages au cours du développement post-embryonnaire. Au microscope électronique à balayage, ces ornementations épicuticulaires apparaissent sous la forme d'écailles pectinées constituées par la juxtaposition de dents qui sont coalescentes à la base et libres au sommet (pl. 2, fig. 3). Les écailles des ơ sont généralement plus larges que celles des o et possèdent 1 à 2 dents de plus. En dehors de ces aires ornementées, la cuticule des Orchestia ne présente que de nombreuses dépressions (Sellem, 1971).

Dans le cas des Niphargus, Magniez (1966) mentionne des zones d'absorption d'ions argent sur le méropodite et l'ischiopodite des $\mathrm{Gn} 1$ et $\mathrm{Gn} 2$ des ơ et des $q$ de $N$. virei Chevreux, $N$. schellenbergi Karaman, $N$. aquilex Schiödte, ces plages n'étant portées que par les $\mathrm{Gn} 1$ des ot et des $q$ chez N.kochianus kochianus Bate. Les gnathopodes de Gammarus pulex pulex (L.) en sont dépourvus d'après cet auteur. Contrairement à Orchestia, il n'existe pas de dimorphisme sexuel très apparent au niveau des Gn1 et Gn2 de N.virei, N.schellenbergi et Gammarus pulex pulex (pl.4). Chez N.virei et N.schellenbergi, comme chez la plupart des Niphargus, le dimorphisme sexuel porte sur les uropodes 3 qui sont beaucoup plus longs chez les ot que

\footnotetext{
* Laboratoire de Biologie animale et générale, Faculté des Sciences de la Vie et de l'Environment, 6, boulevard Gabriel, 21-DIJON
} 
chez les $\&$ (Chevreux et Fage, 1925); chez les Gammares du groupe pulex, les of possèdent non seulement une brosse de soies sur le flagelle des A 2, mais pédoncule et flagelle de ces mêmes antennes sont beaucoup plus épaissis (Roux, 1967). Jusqu'à Magniez (1966), la localisation d'ornementations sur les gnathopodes de Gammaridés n'est précisée que dans quatre cas. En 1893, Della Valle représente une large plage ornementée sur le méropodite des péréiopodes 1 d'Eriopisa elongata. Dans Schellenberg (1942), le méropodite des Gn1 des ot de Synurella ambulans présente (sur le dessin) une plage sternale pourvue d'aspérités et la diagnose de Microniphargus leruthi Schellenberg 1934 mentionne sur le carpopodite des Gn1, un lobe hyalin finement granuleux ("hyalinem, fein gerauhtem Hinterlappen). Enfin la sous-espèce $N$.foreli setiferus diffère principalement du type par "le bord sternal de l'article méral des gnathopodes (surtout ceux de la seconde paire) pourvu d'une callosité chitineuse finement velue" (Balazuc, 1954).

Afin de préciser la localisation des territoires ornementés chez les trois espèces qui retiendront notre attention ( $N$. virei, $N$. schellenbergi et Gammarus pulex pulex), nous observons à la loupe binoculaire et au microscope optique les gnathopodes traités ou non au nitrate d'argent vital. La configuration des ornementations, difficilement analysable dans le détail au très fort grossissement du microscope optique, est étudiée au microscope électronique à balayage, ce dernier appareil permettant également de découvrir de nouvelles régions ornementées non révélées par le traitement à l'argent.

\section{I - LES GAMMARIDÉS CONCERNÉS PAR CETTE ÉTUDE}

\section{A - Genre Niphargus}

\section{Niphargus virei Chevreux 1896}

C'est le plus grand Niphargus de la faune française $(38 \mathrm{~mm}$ de la base des antennes au telson) (Husson, 1949 et 1964). Les individus étudiés proviennent de la grotte de Baume-les-Messieurs (Jura) et de la captation d'eau de la ville de Gorze (Moselle). Suivant les stations et notamment à la grotte de Baume-les-Messieurs on constate, comme l'avaient déjà observé Sollaud et Husson (1959), une coloration plus ou moins accentuée des téguments qui apparaissent jaune-orangé.

\section{Niphargus schellenbergi Karaman}

Cette espèce a longtemps été considérée comme une sous-espèce de Niphargus aquilex après avoir été signalée sous le nom de Niphargus puteanus (Chevreux et Fage, 1925). Elle aime les eaux courantes. Ces Niphargus ont été récoltés sous les pierres de la Source de Ste Foy (Val Suzon) et des premiers mètres du ruisseau.

Les Gammaridés dulçaquicoles hypogés que sont les Niphargus vivent dans les eaux à température basse et constante de tout le domaine aquatique souterrain. On les trouve également aux points d'émergence de ces eaux souterraines: sources obscures et fraîches, résurgences et fontaines où leur presence n'est pas nécessairement due à un entraînement passif (Balazuc, 1954; Kureck, 1967). 
L'élevage de Niphargus virei s'effectue à température relativement basse et constante $\left(11^{\circ}\right)$ et à l'obscurité complète, dans des bacs tapissés d'argile de grotte et remplis d'eau riche en sels dissous. Il est également nécessaire d'offrir aux Niphargus des cailloux entre et sous lesquels ils peuvent satisfaire leur thigmotaxie (Husson, 1949, 1950 et 1959). Ces conditions sont facilement réalisées dans des chambres réfrigérées. Des Gammares coupés en deux assurent pour l'essentiel la nourriture de ces Amphipodes.

Chez N.virei comme chez N.schellenbergi, les $\mathrm{Gn} 1$ et $\mathrm{Gn} 2$ des ơ et des $\uparrow$ sont à peu près semblables.

\section{B - Genre Gammarus}

Gammarus pulex pulex (L.) 1758, Gammaridé dulçaquicole épigé, oculé, pigmenté, est commun dans les eaux courantes ou stagnantes; en population dense, il peut être rencontré aussi sous les pierres du rivage ou dans des endroits très humides. Cette espèce a été récoltée à l'épuisette dans le "ruisseau de Mirande", aux environs de Dijon, où elle abonde en toute saison.

Chez un même individu, qu'il soit ơ ou $q$, un dimorphisme existe entre le Gn1 (propodite obpiriforme) et le Gn2 (propodite quadrangulaire). Par contre, les Gn1 sont à peu de chose près semblables chez le ot et la $\uparrow$ (propodite légèrement plus allongé chez le $\delta$ ). Il en est de même pour les Gn2 (bord palmaire transverse pour la $\uparrow$, oblique et un peu concave pour le $\delta^{*}$ ).

\section{II - TECHNIQUES}

\section{A - Nitrate d'argent vital}

Nous avons utilisé la technique au nitrate d'argent vital en procédant comme suit: après un lavage prolongé de 10 minute à l'eau distillée, afin d'éliminer les sels (chlorures) déposés sur la cuticule, les Crustacés sont immergés durant 1 minute dans une solution de nitrate d'argent à $0,2 \%$. Rincés à nouveau à l'eau distillée, les animaux sont plongés 3 à 4 minute dans un bain de révélateur photographique ou exposés à lumière vive. Les gnathopodes sont alors déshydratés et montés au baume du Canada. A la suite de ce traitement, il apparait un noircissement au niveau de certaines aires cuticulaires, celles présentant précisément une ornementation épicuticulaire. Ce noircissement résulte de la diffusion du sel d'argent à travers la cuticule, de la précipitation des ions lourds au contact des chlorures de l'organisme et de leur réduction en argent métallique sous l'action d'un révélateur photographique ou des photons. Les surfaces ainsi mises en évidence doivent être considérées comme étant perméables aux ions argent et non comme ayant une fonction respiratoire (Graf et Magniez, 1969). 


\section{B - Microscope et loupe photoniques}

Les gnathopodes traités au nitrate d'argent et montés au baume du Canada ainsi que des lambeaux de cuticule prélevés au niveau des surfaces ornementées et montés dans de l'alcool-glycérine sont observés au microscope ordinaire ou à contraste de phase. Les surfaces ornementées sont également visibles dans le cas de Niphargus par simple observation, à la loupe binoculaire, de gnathopodes ou de fragments de cuticule desséchés.

\section{C - Microscope électronique à balayage "Stereoscan” (Cambridge S.A.)}

L'utilisation de cet appareil implique une technique de préparation des échantillons qui est maintenant bien connue. Après fixation au liquide de Bouin, à 1' alcool $70^{\circ}$, au fromol neutre ou mieux encore à l'alcool-chloroforme, les Gn1 et Gn2 d'individus adultes ot et $q$ sont, après l'emploi d'un fixateur aqueux, déshydratés progressivement à l'alcool éthylique $70^{\circ}, 95^{\circ}$ et $100^{\circ}$. La phase de dessication de l'appendice nécessitée par les conditions de fonctionnement de cet appareil, entaîne souvent une rétraction et un plissement des régions cuticulaires ornementées du fait qu'elles ne sont pas calcifiées et qu'elles recouvrent un large sinus sanguin. Pour pallier cet inconvénient les appendices sont, après passage à l'alcool éthylique $100^{\circ}$, traités par le toluène et desséchés à ce stade. Les appendices sont alors collés sur les platines porte-objet du "Stereoscan" et recouverts, sous vide, d'une très fine couche d'or. Nous nous sommes efforcés de photographier de face les plages ornementées afin de pouvoir déterminer les dimensions des ornementations de chaque espèce. Cependant des distorsions inévitables de l'image dues à l'inclinaison de l'échantillon rendent les résultats approximatifs.

\section{III - LOCALISATION ET CONFIGURATION DES ORNEMENTATIONS}

\section{A - Chez Niphargus}

\section{1/Localisation de surfaces ornementées}

L'orientation des gnathopodes comme celle des articles est établie selon la règle de Racovitza (1923). On distinguera un bord proximal et un bord distal, une face tergale et une face sternale, une face interne et une face externe.

Les principales plages ornementées se situent toujours, sur les Gn1 et les Gn2 des ơ et des + , au niveau du méropodite, de l'ischiopodite et du basipodite. Elles se localisent comme suit:

a) Chez Niphargus virei (Gn1 et Gn2 ot et $\%)$. Planche $1 *$.

- Sur la face sternale du méropodite, plage médiane $\left(n^{\circ} 3\right)$ plus ou moins arrondie sur les Gn2 des deux sexes et plus ou moins triangulaire sur les Gn1 des deux

\footnotetext{
* Chez Niphargus et Gammarus, les plages ornementées sont numérotées de 1 à 5 en allant du propodite au basipodite.
} 

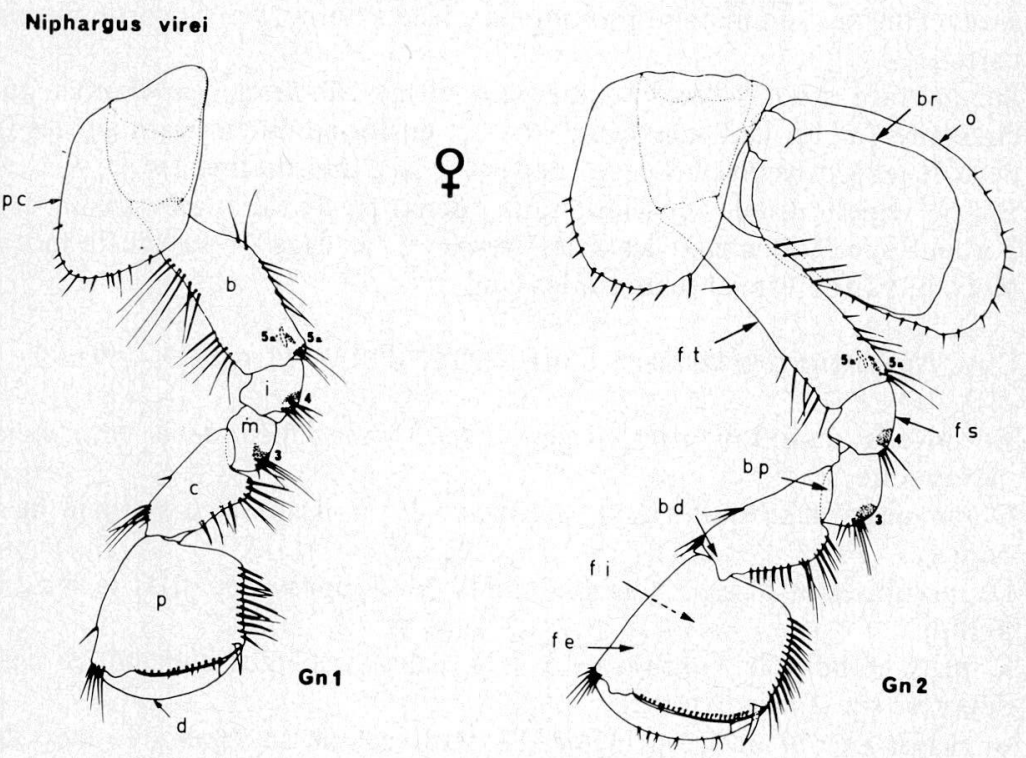

\section{Niphargus schellenbergi}
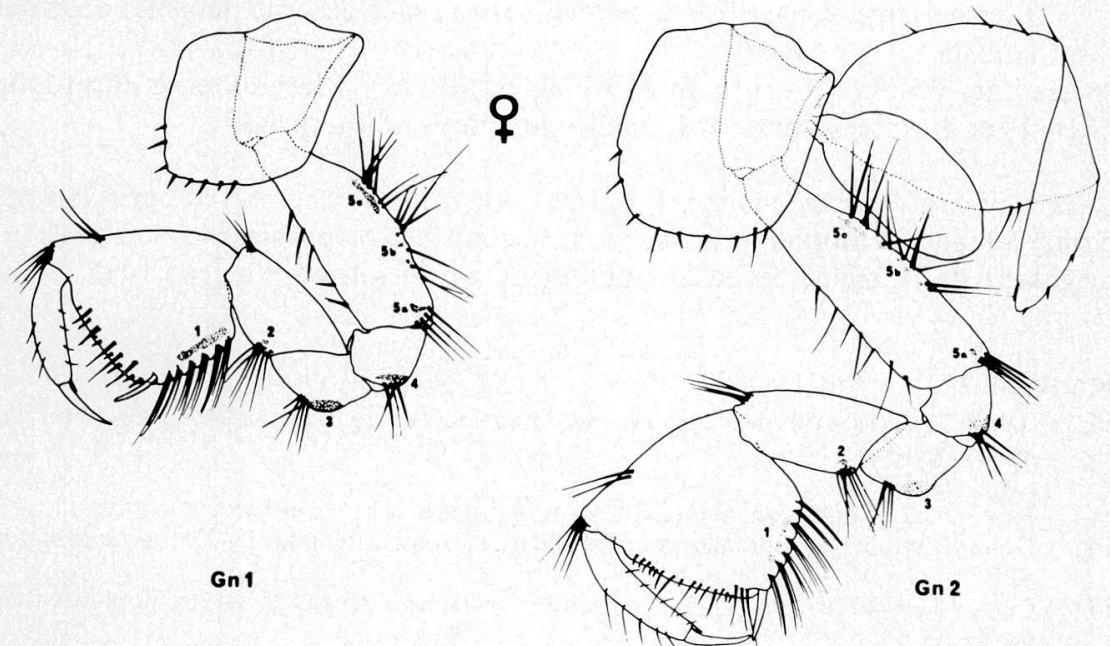

\section{PLANCHE 1}

Localisation des surfaces ornementées sur le Gn1 et le Gn2 de Niphargus virei $\$$ et Niphargus schellenbergi .

$\mathrm{b}=$ basipodite $; \mathrm{bd}=$ bord distal $; \mathrm{bp}=$ bord proximal $; \mathrm{br}=$ branchie $; \mathrm{a}=$ carpopodite $; \mathrm{d}=$ dactylopodite $; \mathrm{fe}=$ face externe; $\mathrm{fi}=$ face interne; $\mathrm{fs}=$ face sternale; $\mathrm{ft}=$ face tergale; $\mathrm{i}$ $=$ ischiopodite $; \mathrm{m}=$ méropodite $\mathrm{o}=$ oostégite $; \mathrm{p}=$ propodite $; \mathrm{p}=$ plaque coxale. 
sexes, l'un des sommets se prolongeant, face externe, vers la partie proximale de l'article;

- Sur la face sternale de l'ischiopodite, plage subdistale (n॰4) plus ou moins circulaire sur les $\mathrm{Gn} 2$ des deux sexes et en forme de croissant sur les Gn1 des deux sexes (croissant plus développé sur la face interne chez les $\delta$ );

- Enfin, région distale du basipodite, deux plages allongées ( $n^{\circ} 5 a$ et $n^{\circ} 5 a^{\prime}$ ) bordent symétriquement les soies sternales, la plage ( $\left.n^{\circ} 5 a\right)$ de la face externe pouvant parfois être absente sur les Gn2 des $\delta^{\circ}$.

b) Chez Niphargus schellenbergi (Gn1 et Gn2 of et 9 ). Planche 1 - Planche 2, fig. 1 et 2 .

- Au niveau d'un lobe très accentué $(n \circ 3)$ au milieu de la face sternale du méropodite;

- D'une plage subdistale (n॰4) en forme de croissant face sternale de l'ischiopodite;

- De nombreuses plages, plus ou moins développées, réparties le long du basipodite:

- plage subdistale $\left(n^{\circ} 5 \mathrm{a}\right)$ de 3 à 4 cellules le plus souvent et légèrement déportée sur la face externe;

- plages $\left(n^{\circ} 5 b\right)$ de 2 ou même d'1 seule cellule soulignant la base des soies médio-sternales;

- enfin une plage (n॰5c) assez importante, de 6 à 8 cellules n'excédant pas 2 à 3 cellules de large, longeant latéralement, sur la face externe, les soies de la partie proximale.

- Chez les $\$$ uniquement, il en existe également sur la face externe du propodite $\left(n^{\circ} 1\right)$ et du carpopodite $\left(n^{\circ} 2\right)$, en bordure des soies sternales.

Chez N.virei et N.schellenbergi, les plages ornementées bien développées du méropodite et de l'ischiopodite, et celles beaucoup plus modestes des autres articles, réagissent de la même façon au traitement par le nitrate d'argent vital. La forte

\section{PLANCHE 2}

Fig. 1 et 2. Localisation des surfaces ornementées $(\not)$ sensibles à $\mathrm{Ag} \mathrm{NO}_{3}$ vital chez N.schellenbergi:

Fig. 1. Gn1 o.

Fig. 2. Gn2 $\%$. Lobe ornementé médio-sternal très accentué sur le métropodite.

Fig. 3 Ornementations épicuticulaires en écaille du carpopodite du $\mathrm{Gn} 1$ d'Orchestia gammarella o.

Fig. 4 Cuticule en dehors des plages ornementées sensibles à $\mathrm{Ag} \mathrm{NO}_{3}$ vital; Ischiopodite du $\mathrm{Gn} 1$ de $N$.virei ơ.

Fig. 5 Ornementations épicuticulaires de la région distale de l'ischiopodite du Gn1 de N.virei o. Fig. 6 Ornementations épicuticulaires du méropodite du $\mathrm{Gn} 1$ de $N$.virei ơ.

$\mathrm{b}=$ basipodite $\mathrm{c}=$ carpopodite $; \mathrm{d}=$ dactylopodite $; \mathrm{di}=$ digitation $; \mathrm{d} \mathrm{p}=$ dépression $; \mathrm{i}=$ ischiopodite; lce $=$ limites des cellules épidermiques; $\mathrm{m}=$ méropodite; $\mathrm{ma}=$ mamelon; $\mathrm{p}=$ propodite.

N.B. Sur les planches photos 2, 3, 5, 6, l'échelle est donnée en microns. 

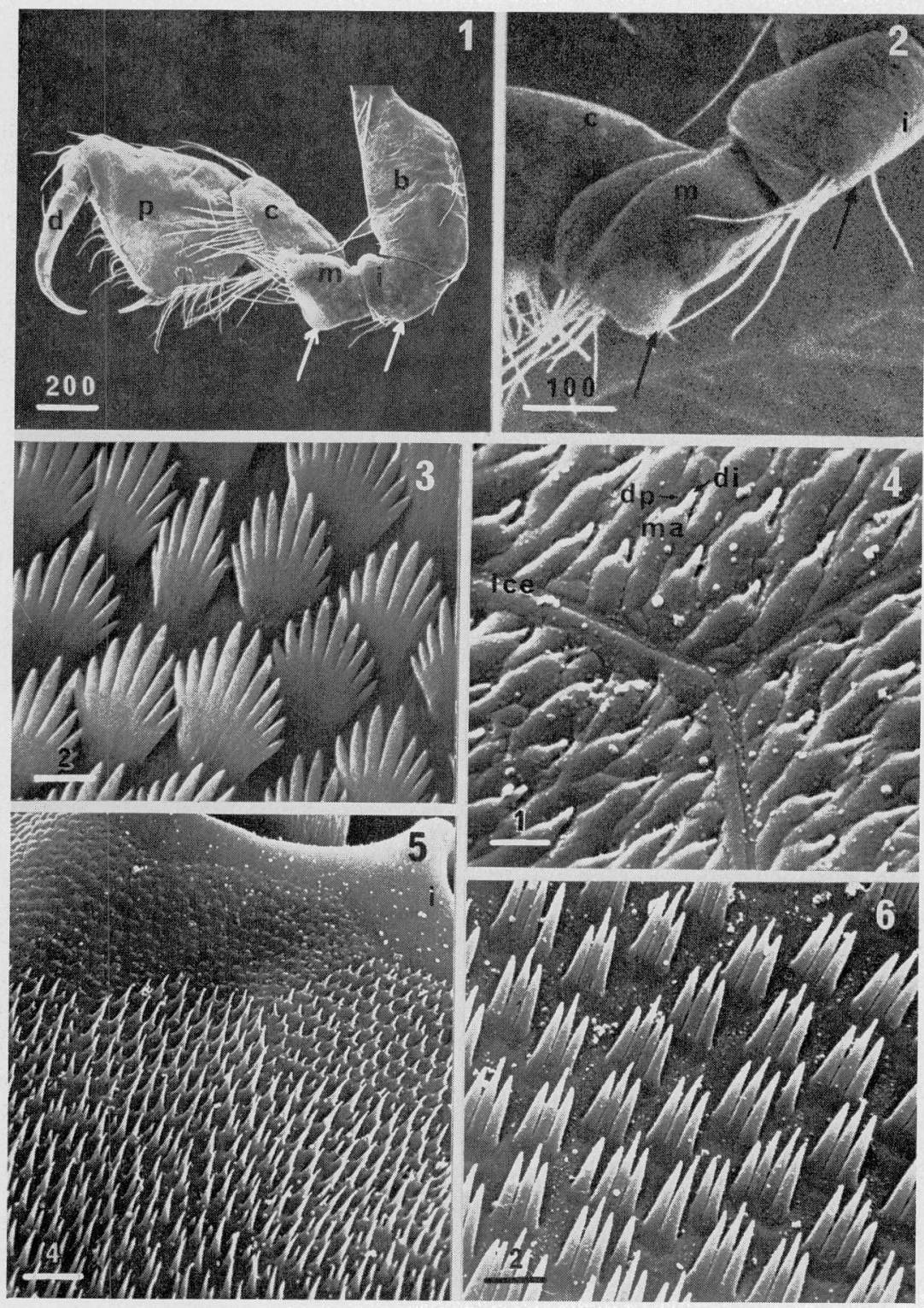
intensité de cette coloration peut être comparée à celle des territoires portant des écailles chez Orchestia.

\section{2/Cuticule en dehors des plages ornementées sensibles au nitrate d'argent vital}

En dehors des surfaces ornementées décelables après réaction au nitrate d'argent vital, le reste de la cuticule des gnathopodes (exception faite pour un réseau polygonal de travées correspondant aux limites des cellules épidermiques et une bande très étroite à la base des soies) présente aussi une ornementation mais qui ne peut être observée qu'au microscope électronique à balayage. A l'intérieur de chaque polygone, le tégument est légèrement mamelonné. Ces mamelons ovoïdes, disposés régulièrement en quinconce et orientés vers le bord distal de chaque article, se terminent par une petite digitation recouvrant une dépression de $0,2 \mu$ environ (pl.2, fig. 4); on en dénombre 300 à 380, selon les cellules, chez N.virei par exemple.

\section{3/Configuration des ornementations}

L'observation des ornementations des aires cuticulaires bien développées sur le Gn 1 ou le Gn2 d'une $\$$ ou d'un $\delta$, au niveau du méropodite, de l'ischiopodite et du basipodite révèle que leur configuration présente une certaine unité: chaque ornementation, toujours orientée vers la région distale de l'article (pl.2, fig.5), est constituée par le groupement de 3 dents essentiellement chez N.schellenbergi alors que chez $N$.virei il y a de 1 à 5 dents avec prédominance de groupement par 3 . Ces digitations, bien individualisées jusqu'à leur insertion sur la cuticule, restent toujours indépendantes chez N.virei (pl.2, fig.6) alors qu'elles sont jointives sur une partie ou la totalité de leur longueur chez N.schellenbergi (pl.3, fig.4). Chez cette espèce, elles ne seront séparées que dans le cas des plages peu développées du basipodite ( $\mathrm{pl} .3$, fig. 1 et 5 ) et au niveau des ornementations du propodite et du carpopodite des $\$$ où il sera alors difficile de les regrouper par 3.

La longueur des ornementations varie selon l'article considéré et l'importance de la plage ornementée: de 3 à $4 \mu$ pour la ou les dents centrales des formations du méropodite, de l'ischiopodite et des grandes plages du basipodite (pl.2, fig.6; pl.3, fig.4), elle n'est plus que de 1 à $2 \mu$ pour les plages isolées du basipodite (pl.3, fig. 1 et 5), de 2,5 à $0,3 \mu$ au niveau du propodite des $\$$ et enfin de 0,5 à $0,3 \mu$ sur le carpopodite des \%. Si les ornementations caractéristiques ont une longueur à peu

\section{PLANCHE 3}

Fig. 1.Plage ornementée (n॰5b) soulignant les soies médio-sternales du basipodite du $\mathrm{Gn} 2$ de N.schellenbergi ơ.

Fig. 2.Densité des ornementations au niveau de l'ischiopodite du Gn1 de N.virei o. Limites des cellules épidermiques dessinant un réseau polygonal très net.

Fig. 3. Basipodite du Gn1 de N.virei o: passage progressif de la plage à ornementations constituées par le groupement de dents vers la cuticule mamelonnée.

Fig. 4.Densité des ornementations au niṿeau du méropodite du Gn1 de N.schellenbergi o. Limites cellulaires soulignées.

Fig. 5. Ornementations du basipodite (plage n॰5c) du $\mathrm{Gn} 2$ de N.schellenbergi ơ.

Fig. 6. Bord palmaire du Gn2 ot de N.virei.

$\mathrm{bpa}=$ bord palmaire $; \mathrm{p}=$ propodite . 

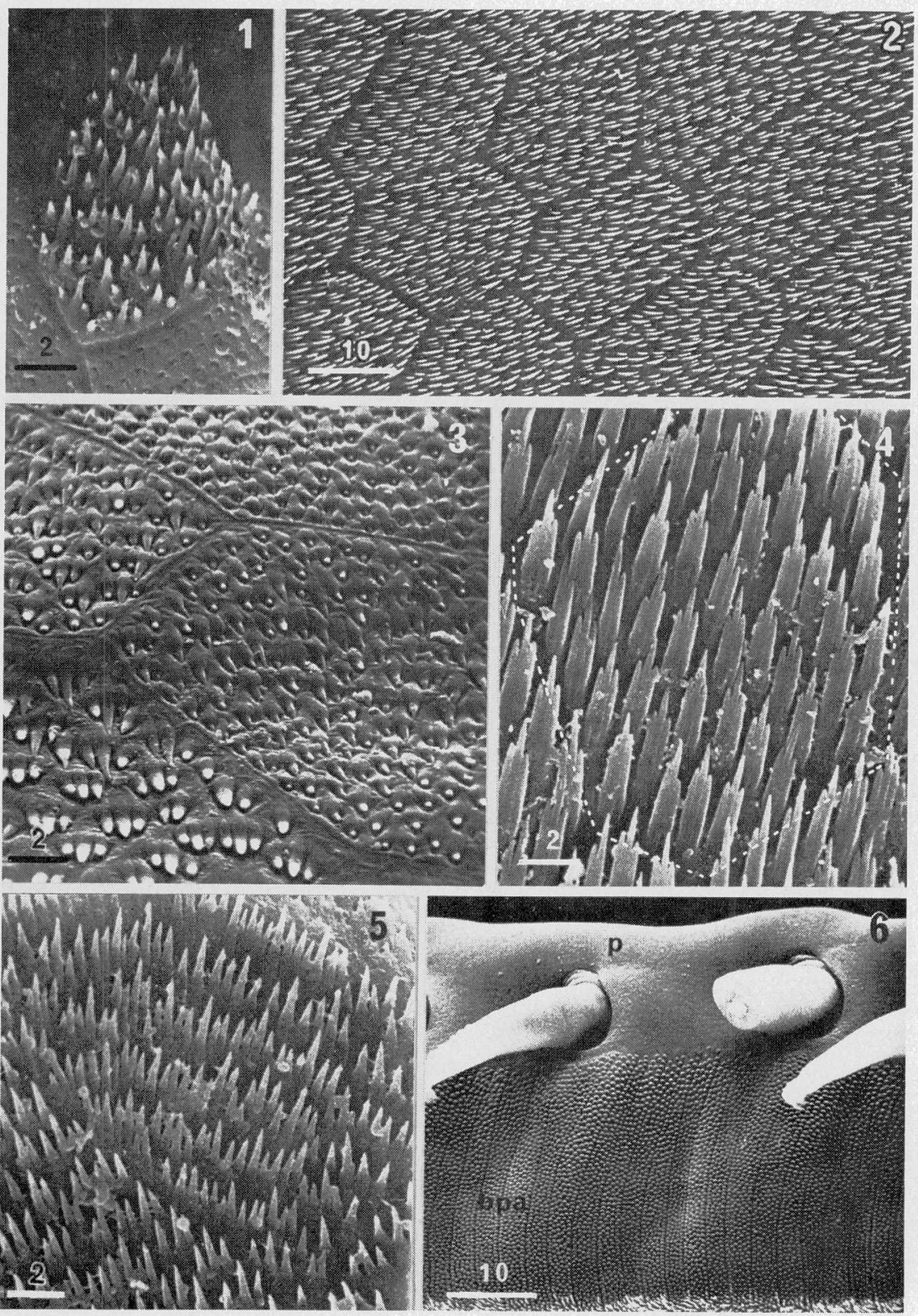
près constante chez les deux espèces observées, leur largeur varie de 1,5 à $2,5 \mu$ selon le nombre de dents chez $N$.virei (pl.2, fig.6) alors qu'elle ne dépasse pas $1 \mu$ chez N.schellenbergi ( $\mathrm{pl} .3$, fig.4).

Disposées généralement en quinconce (pl.2, fig.6) les ornementations semblent s'aligner sur des arcs de cercle à faible rayon de courbure au niveau des plages peu étendues du basipodite (pl.3, fig.5). On ne retrouve plus aucune figure caractéristique sur le propodite et encore moins sur le carpopodite des $\uparrow$ de N.schellenbergi.

$\mathrm{Au}$ sein d'une plage, les formations épicuticulaires sont groupées en polygones dont les contours correspondent aux limites des cellules épidermiques (pl.3, fig.2) et à l'échelle cellulaire, la présence ou l'absence de formations répondent à la loi du tout ou rien ( $\mathrm{pl} .2$, fig. 5 ; pl.3, fig.1), c'est à dire que toute la surface cuticulaire sus-jacente à une cellule est ornementée ou ne l'est pas.

On constate un passage très progressif entre les cellules à ornementations denticulées et celles dont la cuticule est mamelonnée. En effet, les dents latérales se réduisent tout d'abord, puis la ou les dents centrales se raccourcissent (pl.2, fig.5). Il semble que ces dernières ne disparaissent jamais complètement car, comme nous l'avons déjà mentionné, il persiste une digitation à la partie distale des mamelons de toute la surface cuticulaire des gnathopodes (pl.3, fig.3).

La densité des ornementations par unité de surface c'est-à-dire leur nombre par polygone est variable selon l'importance de la plage ornementée (pl.3, fig.1 et 4). Pour des cellules de 17 à $20 \mu$ sur 12 à $15 \mu$ en moyenne chez N.schellenbergi et de 20 à $24 \mu$ sur 15 à $19 \mu$ chez $N$. virei, nous comptons environ 50 formations chez les premiers et 60 chez les seconds (pl.3, fig. 2 et 4 ).

En dehors de ces ornementations, les Gn1 et Gn2 des ot et des $\$$ possèdent d'autres types de formations épicuticulaires. De part et d'autre de l'arête du bord palmaire pourvue d'ornementations aciculaires, on observe des mamelons disposés en quinconce chez N.schellenbergi et de plus, groupés en bandes parallèles chez N.virei (pl.3, fig.6).

Ainsi, les gnathopodes de ces deux espèces ne présentent pas de dimorphisme sexuel et le sexe des individus n'influence pas la configuration des ornementations. N.schellenbergi possède des plages ornementées supplémentaires sur le propodite et sur le carpopodite des $\mathrm{Gn} 1$ et des $\mathrm{Gn} 2$ des 9 . Les ornementations de N.virei ont une longueur plus homogène et un nombre de dents plus variable que celles de N.schellenbergi.

\section{B - Chez Gammarus pulex pulex}

\section{1/Localisation des surfaces ornementées}

Les principales plages ornementées se situent au niveau du méropodite, de l'ischiopodite et du basipodite comme chez Niphargus, mais également sur le carpopodite des 9 . Elles se localisent comme suit:

a) ơ adulte ( $\mathrm{Gn} 1$ et $\mathrm{Gn} 2)$ - Planche 4.

- Sur la face sternale du méropodite, grande plage médiane $\left(\mathrm{n}^{\circ} 3\right)$ plus ou moins triangulaire dont l'un des sommets s'étend, face externe, vers la région proximale de l'article; 


\section{Gammarus pulex pulex}

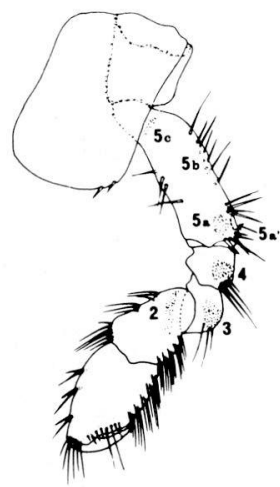

Gn 1
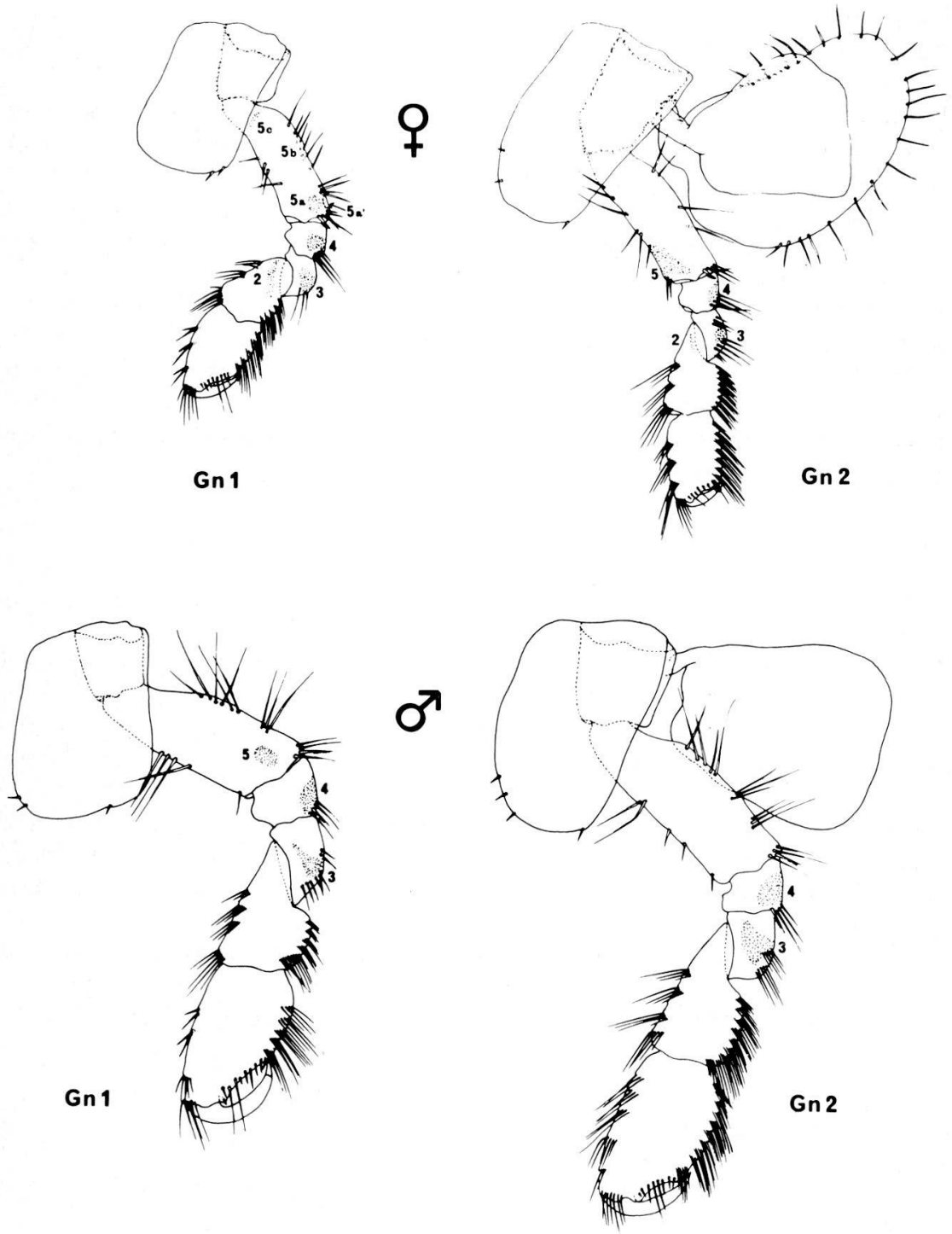

PLANCHE 4

Localisation des surfaces ornementées sur le Gn1 et le Gn2 de Gammarus pulex pulex $\%$ et $\delta$. 
- Sur la face sternale de l'ischiopodite, plage (n०4) en forme de large croissant recouvrant une grande partie de l'article (particulièrement sur le Gn2);

- Enfin, région subdistale du basipodite des Gn1 seulement, plage unique (n5) sur la face externe.

b) i adulte - Planche 4 .

Gnl

- Sur la face sternale du méropodite et de l'ischiopodite, plages médianes ( $n^{\circ} 3$ et $\left.n^{\circ} 4\right)$ plus ou moins arrondies se prolongeant en une pointe, sur la face interne, vers la région proximale de chaque article;

- Sur le basipodite:

- deux plages subdistales $\left(n^{\circ} 5 a_{\text {e }} n^{\circ} 5 a^{\prime}\right)$ plus ou moins arrondies sur les faces externe et interne;

- de petites plages réduites $\left(n^{\circ} 5 b\right)$ soulignant la base des soies sternales de la région médiane;

- une plage proximale ( $\mathrm{n}^{\circ} 5 \mathrm{c}$ ) longeant, sur la face externe, le bord de la plaque coxale.

- Enfin, sur la face externe du carpopodite, plage proximale $\left(n^{\circ} 2\right)$ allongée bordant la région distale du méropodite.

Gn2

- Sur la face sternale du méropodite et de l'ischiopodite, plages médianes ( $n^{\circ} 3$ et n०4) en forme de croissant;

- Pour la première fois, plage subdistale $\left(n^{\circ} 5\right)$ très développée sur la face tergale du basipodite;

- Eventuellement, dans la région proximale du carpopodite, plage ornementée $\left(n^{\circ} 2\right)$ sur la face externe.

Dans des conditions d'expérimentation identiques à celles employées pour Niphargus et Orchestia, seules les ornementations médio-sternales du méropodite ( $n \circ 3$ ) et de l'ischiopodite (n॰4) de Gammarus pulex pulex se colorent très faiblement après traitement au nitrate d'argent vital. Si nous augmentons la concentration du réactif ou la durée de l'immersion, toute la cuticule se teinte en brun et la limite des plages est alors difficilement discernable.

Comme chez Niphargus, le reste du tégument des gnathopodes est recouvert de mamelons ovoïdes dont l'extrémité distale est terminée par une digitation recouvrant une dépression. Selon les cellules, on peut en compter 230 à 400 .

\section{PLANCHE 5}

Gammarus pulex pulex.

Fig. 1.Plage ornementée médio-sternale de l'ischiopodite du Gn2 o.

Fig. 2 à 5 . Dimorphisme sexuel au niveau des ornementations aciculaires du méropodite:

Fig. 2.Gn1 o.

Fig. 3.Gn1 o.

Fig. 4.Gn2 + .

Fig. 5.Gn2 0 .

Fig. 6.Ornementations aciculaires de l'ischiopodite du $\mathrm{Gn} 2{ }^{\circ}$.

Fig. 7.Ornementations aciculaires du carpopodite du $\mathrm{Gn} 1$. 

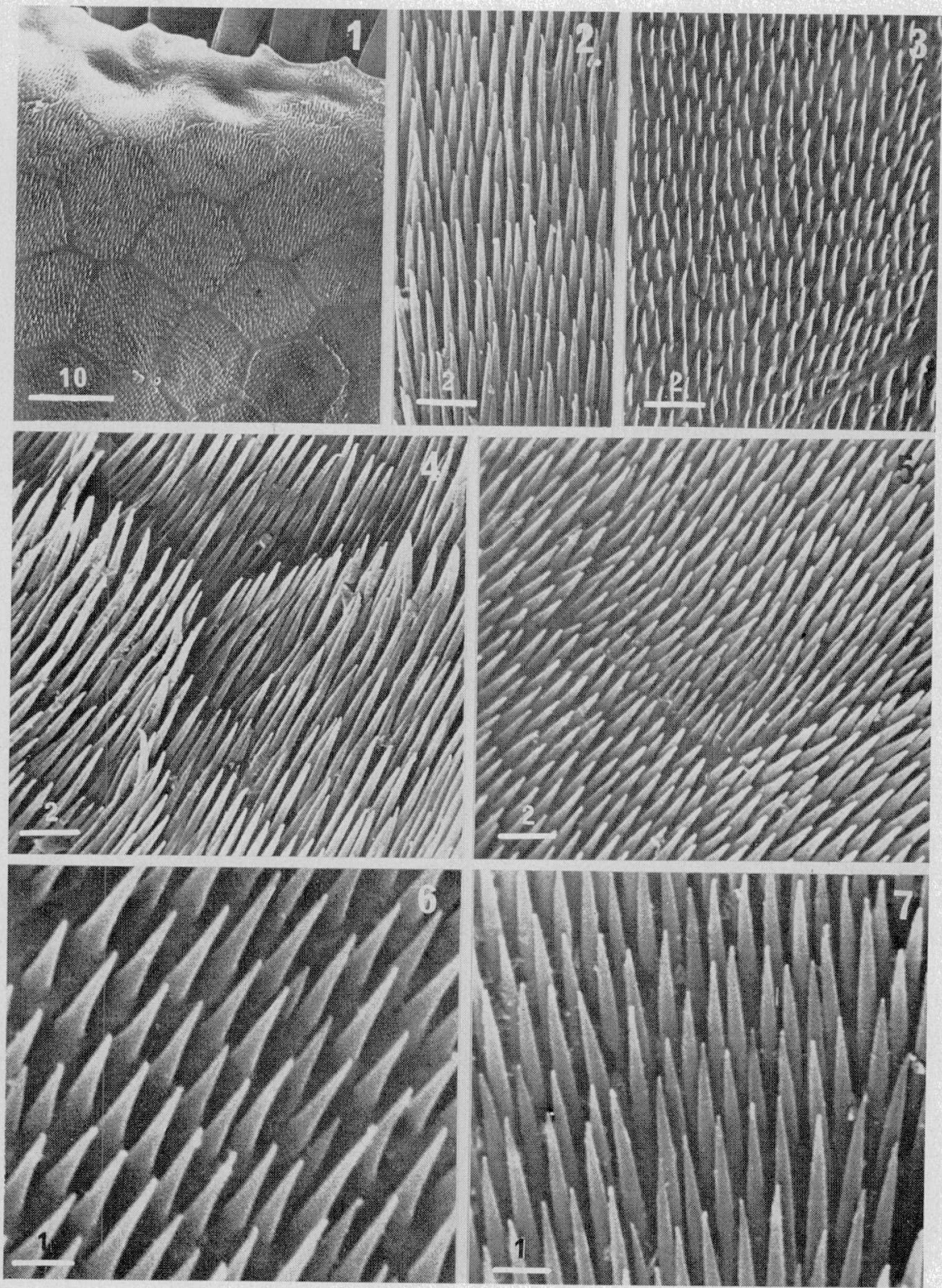


\section{2/Configuration des ornementations}

L'ornementation cuticulaire des gnathopodes de Gammarus diffère de ce qui vient d'être décrit chez Niphargus et de ce qui a été observé chez Orchestia (Graf et Sellem, 1970; Sellem, 1971) par le fait qu'elle est constituée de formations aciculaires (pl.5) qui ne sont, ni groupées, ni coalescentes, mais dont la densité est considérable. Au niveau de l'ischiopodite par exemple (pl.6, fig.5), pour une cellule de $14 \mu$ sur $12 \mu$ environ, elle est de 200 à 240 .

Selon le sexe, l'article et la localisation des plages ornementées sur l'article, nous constatons des variations dans la dimension de ces ornementations:

- L'influence du sexe des individus sur la longueur des ornementations aciculaires est particulièrement nette sur les méropodites: de 1 à $2 \mu \mathrm{chez}$ les ơ (pl.5, fig. 3 et 5 ), elles atteignent 3 à $4 \mu$ chez les $\$$ (pl.5, fig.2 et 4 ) tout en conservant une largeur identique $(0,3$ à $0,4 \mu)$ au niveau de leur insertion sur la cuticule.

- Sur les plages bien développées de l'ischiopodite (pl.5, fig.6), du basipodite ( ${ }^{\circ} 5$, $\mathrm{n}^{\circ} 5 \mathrm{a}, \mathrm{n}^{\circ} 5 \mathrm{a}^{\prime}$ ) et du carpopodite (pl.5, fig.7), les ornementations ont une longueur d'environ 1 à $2 \mu$ sauf pour quelques dents de 3 à $4 \mu$ au niveau du basipodite (pl.6, fig.1) alors qu'elles sont très petites et mesurent exceptionnellement plus de $1 \mu$ (pl.6, fig. 2 et 3 ) sur les autres plages du basipodite ( $\left.n^{\circ} 5 \mathrm{~b}, \mathrm{n}^{\circ} 5 \mathrm{c}\right)$ et du carpopodite des $\mathrm{Gn} 2$ des $\uparrow$, quand elles existent.

Les ornementations recouvrent chaque polygone en totalité. Implantées en quinconce (pl.5) sur le carpopodite des Gn1 des + , le méropodite et l'ischiopodite, les formations aciculaires semblent s'aligner plus ou moins régulièrement sur les autres articles (pl.6, fig.1 et 2), exception faite pour les plages ( ${ }^{\circ} 5 b$ et $\left.n^{\circ} 5 c\right)$ du basipodite des Gn1 des 9 qui ne montrent aucune répartition préférentielle (pl.6, fig. 3).

Sur le pourtour des plages ornementées, les dents sont plus courtes, renflées à leur base et alignées en de larges festons (pl.6, fig.4). Sous l'extrémité distale de chacune de ces dents, la cuticule est déprimée, ce qui indique un passage progressif vers le tégument des gnathopodes orné de mamelons ovoïdes associés à des dépressions.

Quant au bord palmaire (pl.6, fig.6), il ne présente pas de formations aciculaires mais uniquement de nombreux mamelons regroupés en bandes parallèles.

Ainsi, la répartition des plages est différente, non seulement sur le basipodite des $\mathrm{Gn} 1$ et des $\mathrm{Gn} 2$ présentant un dimorphisme chez un même individu qu'il soit ơ ou $\uparrow$, mais également sur chacun de ces appendices pris cette fois dans les deux sexes.

\section{PLANCHE 6}

Gammarus pulex pulex. Ornementations épicuticulaires aciculaires (fig. 1 à 5), en mamelons (Fig. 6):

Fig. 1.Basipodite (plage tergale $\mathrm{n}^{\circ} 5$ ) du $\mathrm{Gn} 2$ \%.

Fig. 2.Carpopodite du Gn2 .

Fig. 3.Basipodite (plage $\mathrm{n}^{\circ} \mathrm{5c}$ ) du $\mathrm{Gn} 1$ \%.

Fig. 4.Méropodite du Gn1 $\delta$ : sur le pourtour de la plage, les ornementations diminuent de longueur et se renflent à leur base.

Fig. 5.Ischiopodite du Gn2 . Densité des ornementations considérable par rapport à Orchestia et Niphargus.

Fig. 6.Bord palmaire du Gn1 o. 

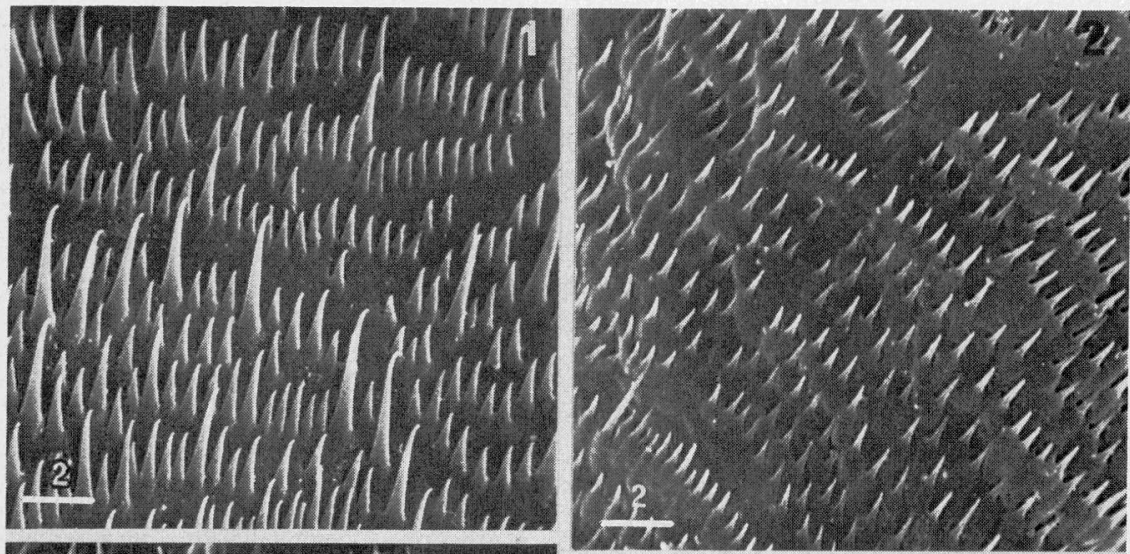

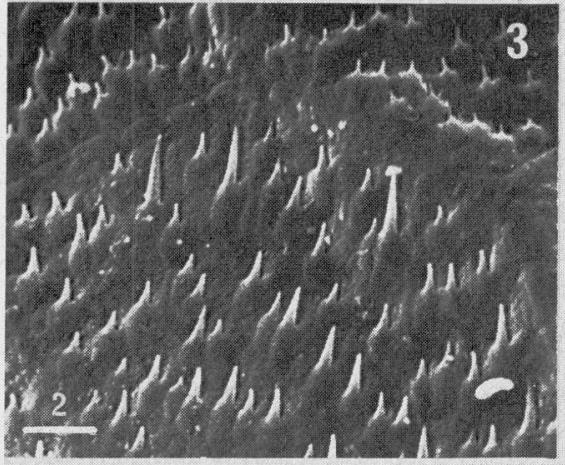
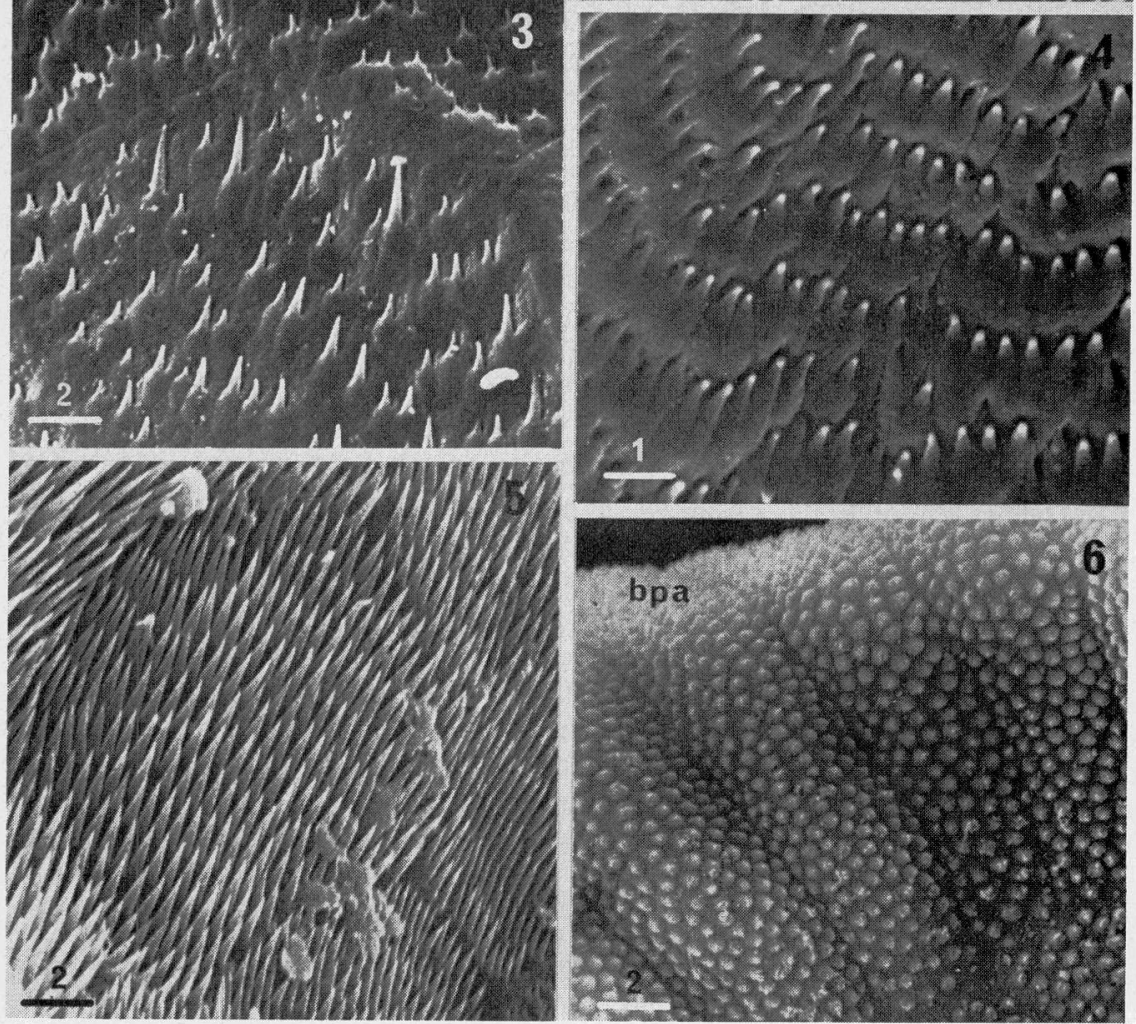
En effet, on note une série de plages ornementées sur la basipodite des $\mathrm{Gn} 1$ des $q$, alors qu'on n'en dénombre une seule sur celui des $\mathrm{Gn} 1$ des o; de même sur le basipodite des $\mathrm{Gn} 2$ des $\uparrow$ on observe pour la première fois une plage ornementée sur la face tergale tandis que celui du Gn2 des ơ ne présente aucune formation.

Les carpopodites des Gn1 et des Gn2 des $q$ de Gammarus pulex pulex possèdent des plages ornementées que l'on ne retrouve pas chez les $\delta$.

Enfin, un dimorphisme sexuel très net apparaît au niveau des ornementations des plages bien développées du méropodite: les dents des ơ ( 1 à $2 \mu)$ sont beaucoup moins longues que celles des $\subsetneq(3$ à $4 \mu)$.

\section{IV - CONCLUSION}

Le microscope électronique à balayage nous a permis d'établir que Gammarus pulex pulex possède sur les $\mathrm{Gn} 1$ et $\mathrm{Gn} 2$ des ot et des $q$ des plages ornementées d'importance inégale mais dont les principales sont situées sur le méropodite et l'ischiopodite de la même façon que chez Niphargus. Cette localisation diffère totalement de celle rencontrée chez Orchestia.

L'étude de ces régions particulières montre qu'elles sont pourvues d'une ornementation épicuticulaire qui est caractéristique de chacun des genres observés: ensemble de trois dents, jointives sur une partie ou la totalité de leur longueur chez $N$. schellenbergi; libres jusqu'a leur insertion sur la cuticule chez N.virei; ornementations toutes aciculaires chez Gammarus pulex pulex. Il semble cependant que la configuration très dissemblable de l'ornementation n'est qu'apparente et que cette ornementation est toujours constituée de formations aciculaires qui restent simples chez Gammarus, qui se groupent le plus souvent par trois chez Niphargus et qui s'associent en plus grand nombre chez Orchestia en constituant des écailles pectinées. Ainsi, l'ornementation de N.schellenbergi et de N.virei apparaît comme étant un moyen terme entre celle de Gammarus pulex pulex, très simple, et celle beaucoup plus élaborée des Orchestia étudiées.

Si l'on considère alors comme élément de base une formation aciculaire de Gammare, la densité des ornementations par cellule qui est de 220 chez Gammarus pulex pulex devient égale à $150(50 \times 3)$ chez N.schellenbergi et à $180(60 \times 3)$ chez N.virei. Si l'on prend comme référence la surface d'une cellule épidermique de Gammarus pulex pulex, pour cette même surface il y a en moyenne 100 formations élémentaires chez N.schellenbergi et $80 \mathrm{chez}$ N.virei. Nous constatons ainsi que la densité des éléments est beaucoup plus forte chez Gammarus pulex pulex que chez $N$.virei et $N$.schellenbergi où elle est sensiblement égale malgré la grande différence de taille de ces deux dernières espèces. La densité des ornementations d'Orchestia serait assez semblable à celle des Niphargus.

Si l'on ne s'occupe que des caractères liés au sexe des individus étudiés, on s'aperçoit que:

1) Chez N.schellenbergi et Gammarus pulex pulex, le ơ possède moins de plages ornementées sur ses gnathopodes que la $q$, ce qui est à rapprocher du cas des Orchestia. Cependant ce fait est dû à des raisons différentes: 
- chez N.schellenbergi et Gammarus pulex pulex, c'est une question de plages supplémentaires puisque la $q$ présente seule des ornementations sur le carpopodite ou sur le carpopodite et le méropodite.

- chez Orchestia, par contre, c'est une question de surface totale des formations épicuticulaires: celle des ot adultes (plus de 17 articles au flagelle des A 2) correspond au $1 / 3$ de celle des $q$ du même stade.

2) En ce qui concerne leur configuration sur les méropodites, les dents simples de Gammarus pulex pulex présentent un dimorphisme sexuel: elles sont plus longues chez les $q$ que chez les $\delta$, alors que chez Orchestia toutes les écailles des différents articles ornementés sont plus larges chez les ot que chez les + .

En dehors de ces régions ornementées, la surface cuticulaire des gnathopodes est semblable chez les deux Gammaridés Niphargus et Gammarus mais différente de celle des Orchestia qui ne présente que des dépressions réparties sans ordre défini à l'intérieur des contours cellulaires.

\section{RESUME}

Les Gn1 et Gn2 de Niphargus virei, N.schellenbergi (Gammaridés hypogés) et Gammarus pulex pulex (Gammaridé épigé) ot et $q$ possèdent des plages ornementées que nous avons étudiées au microscope électronique à balayage. Les ornementations sont constituées de dents. Elles sont simples chez Gammarus pulex pulex et celles du méropodite présentent un dimorphisme lié au sexe. Elles se regroupent le plus souvent par trois chez N.schellenbergi et N.virei. Chez Orchestia (Talitridé), les dents se soudent à leur base en formant des écailles pectinées qui montrent également un dimorphisme sexuel.

En dehors de ces régions particulières, la cuticule des gnathopodes de N.virei, N.schellenbergi et Gammarus pulex pulex est ornée de mamelons ovoïdes prolongés par une digitation recouvrant une dépression.

\section{SUMMARY}

Niphargus virei and N.schellenbergi (hypogean Gammarids) as also Gammarus pulex pulex (epigean Gammarid) show on the Gn1 and $\mathrm{Gn} 2$ of both sexes ornamented areas which were studied with a scanning electron microscope. The ornamentations are built up of teeth. These are simple in Gammarus pulex pulex and present a sexual dimorphism on the meropodite. In N.schellenbergi and N.virei they are as often as not grouped by three. In Orchestia (Talitrid) the teeth have their bases fusioned which forms pectinate scales showing a sexual dimorphism.

Outside these areas, the gnathopod cuticle of N.virei, N.schellenbergi and Gammarus pulex pulex is ornamented with ovoid papilla, each of them surmounted by a finger-like process covering over a depression. 


\section{BIBLIOGRAPHIE}

BALAZUC, J., 1954 - Les Amphipodes troglobies et phréatobies de la faune gallo-rhénane. Arch. Zool. expér. gén., Fr., 91, 153-193.

CHARNIAUX-COTTON, H., 1957 - Croissance, régénération et déterminisme endocrinien des caractères sexuels d'Orchestia gammarella (Pallas) Crustacé Amphipode. Ann. Sci. nat., Fr., 19, 411-560.

CHEVREUX, E. et FAGE, L., 1925 - Amphipodes. Faune de France, 9, Lechevalier, Paris, $1-488$.

DELla VAlLE, A., 1893 - Gammarini del Golfo di Napoli. Zool. Stat. Z. Neapel, Fauna und Flora, 20. Mon., Friedländer, Berlin.

GRAF, F. et MAGNIEZ, A., 1969 - Surfaces respiratoires, aires cuticulaires perméables aux sels (nitrate d'argent et leuco-dérivés) et régions squelettiques non calcifiées $\mid$ chez quelques Crustacés Amphipodes. C. R. Acad. Sci., Fr. 268, 3191-3194.

GRAF, F. et SELLEM, E., 1970 - Les formations épicuticulaires des gnathopodes d'Orchestia (Crustacé, Amphipode): étude morphologique et topographique au cours du développement post-embryonnaire. C. R. Acad. Sci., Fr., 270, 1472-1475.

HUSSON, R., 1949 - Observations biologiques sur l'Amphipode Niphargus virei Chev. (Note préliminaire). Bull. Soc. zool., Fr., 74, 126-131.

HUSSON, R., 1950 - Observations biologiques sur l'Amphipode Niphargus virei Chev. (deuxième note). Bull. soc. zool., Fr., 75, 211-217.

HUSSON, R., 1959 - Les Crustacés Péracarides des eaux souterraines. Considérations sur la biologie de ces cavernicoles. Bull. Soc. zool., Fr., 84, 219-231.

HUSSON, R., 1964 - Considérations sur la taille des troglobies aquatiques. Troisième Congrès international de Spéléologie. Wien, 71-74.

KURECK, A., 1967 - Über die tagesperiodische Ausdrift von Niphargus aquilex schellenbergi Karaman aus Quellen. Z. Morph. Ökol. Tiere, 58, 247-262.

MAGNIEZ, A., 1966 - Recherche des surfaces respiratoires et des zones cuticulaires perméables aux sels (par l'emploi de nitrate d'argent et de leuco-dérives) chez quelques Crustacés Amphipodes. Dipl. Et. Sup. Sc. nat., Dijon, 1 à 48.

RACOVITZA, E.G., 1923 - Notes sur les Isopodes. 10. Orientation de l'Isopode et essais de nomenclature pratique des bords et faces de son corps et de ses appendices.Arch. Zool. expér. gén., Fr., 61, 75-82.

ROUX, A.L., 1967 - Les Gammares du groupe pulex (Crustacés Amphipodes). Essai de Systématique biologique. Thèse Doct. Sci. nat. Lyon, Arch. orig. Centre Document. C.N.R.S., n०447, 1-147.

SCHELLENBERG, A., 1942 - Krebstiere oder Crustacea. IV: Flohkrebse oder Amphipoda. Tierwelt Deutschlands, 40, Fischer, Iéna, 1-252.

SELLEM, E., 1971 - Contribution à l'étude de l'ornementation cuticulaire des gnathopodes de divers Crustacés Amphipodes. Dipl. Et. Sup. Sc. nat., Dijon, 1 à 73.

SELLEM, E. - Utilisation du microscope électronique à balayage dans l'étude systématique des Crustacés Péracarides. (En collaboration avec J.-P. HENRY et G. MAGNIEZ). Note présentée au $97^{e}$ Congrès National des Sociéties Savantes, Nantes, mars 1972, 10 pages dactylographiées, 3 planches. Sous presse. 